\title{
Prediction Model of the Mechanical Behavior of a Fuel Cell Stack under Strengthened Road Vibrating Conditions
}

\author{
Liying Ma $\mathbb{D},{ }^{1,2}$ Bo Lv $\mathbb{D},{ }^{1,2}$ Yongping Hou $\mathbb{D}^{1,2}$ and Xiangmin Pan $\mathbb{D}^{3}$ \\ ${ }^{1}$ Lab of Clean Energy Automotive Engineering Center, Tongji University, Shanghai 201804, China \\ ${ }^{2}$ School of Automotive Studies, Tongji University, Shanghai 201804, China \\ ${ }^{3}$ Shanghai Motor Vehicle Inspection Certification \& Technology Innovation Center, No. 68 Yutian South Road, \\ Shanghai 201805, China \\ Correspondence should be addressed to Yongping Hou; yphou@tongji.edu.cn
}

Received 2 December 2020; Revised 28 June 2021; Accepted 14 July 2021; Published 4 August 2021

Academic Editor: Houari Ameur

Copyright (c) 2021 Liying Ma et al. This is an open access article distributed under the Creative Commons Attribution License, which permits unrestricted use, distribution, and reproduction in any medium, provided the original work is properly cited.

In this paper, a data-oriented model has been presented by nonlinear autoregressive exogenous model (NARX) neural network, which aims at predicting the mechanical behavior of a fuel cell stack for vehicle under the real-life operational conditions. A 300-hour vibration test with reproduction of SVP road spectrum was completed on a Multi-Axial Simulation Table. At the same time, data acquisition of drive displacement and acceleration response on stack was carried out in every 50 hours. All data collected were used to train and evaluate the model based on NARX. Result shows that the prediction model built is of good precision and consistent with the actual situation.

\section{Introduction}

With the development of technology, economy, and people's living standard, the number of automobiles has been increased sharply, which brought about energy shortage and air pollution. Hydrogen is a clean energy with high calorific value which is abundant in earth. Hydrogen fuel cells convert hydrogen into electricity directly with high efficiency, among which proton exchange membrane fuel cell (PEMFC) is most widely used mainly for their wide range of operating temperature and high energy density. Therefore, fuel cell vehicles are going to be one of the main directions of automobile industry in the future.

As a critical component installed on the vehicle, the fuel cell stack is subjected to road excitation passing through the car frame. Exposed to such harsh conditions, it requires the characteristics of mechanical reliability and durability. According to the policy called Made in China 2025 and the US Department of Energy (DOE), lifetime of fuel cell vehicles needs to reach $5000 \mathrm{~h}$ by 2020 [1]. However, now in the laboratory, this target is still on the way. Therefore, study of mechanical vibration performance of fuel cells is an important research subject.

Since 2004, many scholars have been investigating the vibration characteristics of fuel cell stacks. Experimental research and numerical simulation are two commonly used methods. Bétournay et al. [2] rigidly connected a $35 \mathrm{~W}$ fuel cell stack to the chassis of a mine machine, which subjected to vibration and shock for $49 \mathrm{~h}$ in the mine, and tested the performance of the fuel cell stack daily. The result showed that there was no obvious mechanical damage; however, its polarization curve fluctuated obviously at high current densities. Liu et al. [3] investigated the influence of structure and clamping force on PEMFC mode shapes via finite element analysis.

These two research methods mentioned above play an important role in investigating the mechanical characteristics of fuel cell stacks. But it is still a tough work to establish a model with high precision and robustness since the fuel cell system is a complex nonlinear system with many physicochemical parameters that are difficult to determine. Recently, artificial neural network (ANN) model has been widely used with the development of calculation theory and computer 
performance. ANN model, also known as "black box" model, can provide corresponding outputs accurately through the input variables when the input-output mapping relationship is established.

Khatir et al., Tran-Ngoc et al., and Zenzen et al. [4-8] put forward the combination of optimization algorithm and machine learning algorithm, which provided ANN advantages of fast convergence and global search, and solved a series of engineering structure damage identification problems. Their research results expanded the research ideas of artificial neural network optimization algorithm and its application scenarios in engineering.

Jr et al. [9] employed dynamic neural networks to build a prediction model based on time series data. Feed forward time delay neural network (TDNN), Elman neural network, and NARX neural network were adopted and their forecasting capabilities were compared. Two real-world datasets were used, namely, chaotic laser time series and a variable bit rate (VBR) video traffic time series. They reported that NARX neural network with the feedback loop of delayed outputs did much better job in prediction than that of TDNN and Elman networks.

Devi et al. [10] compared the performance of ANN models for rainfall time series data prediction. Several ANN models, such as feed forward back propagation neural network (BPN), cascade forward back propagation neural network (CBPN), distributed time delay neural network (DTDNN), and NARX network, were adopted to predict rainfall datasets in Nilgiris and Coonoor. From observed mean square error (MSE) and correlation coefficient (CC) values which were used as two performance indicators, NARX network outperformed all the other networks.

Rouss [11-14] proposed a new modeling approach for fuel cell stack, which is of complex nonlinear nature. They investigated the mechanical characteristics of fuel cell stacks under vibrating conditions in aircraft application from two parts: experiment and modeling. Firstly, they set up a vibration test bench. Then, they tested a 5-cell fuel cell stack for all three orthogonal axes using a swept sine signal with a frequency bandwidth from 6 to $2000 \mathrm{~Hz}$ and acceleration amplitude from 1 to $20 \mathrm{~g}$. The response acceleration of the stack was measured by a three-dimensional (3D) accelerometer. After that, a gas tightness test was conducted to ensure the fuel cell stack was in normal working conditions. When it came to modeling, they proposed a multi-input multioutput (MIMO) model using a multilayer perceptron neural network. The ANN model was tested after training and pruning. The results showed that the model is of good precision accuracy and thus can be extended to further applications, such as fault diagnosis.

Literatures mentioned above verify the superiority of NARX neural network in dealing with different kinds of time series datasets and investigate the mechanical characteristics as well as modeling of aircraft fuel cell stacks under dynamic loads. However, a model that predicts the mechanical behavior of a fuel cell stack in vehicle applications under strengthened road vibrating conditions has not appeared yet.

In this paper, a prediction model of vehicle fuel cell stack based on NARX neural network is established. The actual excitation was measured and divided into training sets and validation sets. Then, the model was trained by the training sets; then, the prediction performance was validated from the following three aspects: the contrast analysis for network outputs and target values in both time domain and frequency domain, the correlation test, and the eigenvalue test.

\section{Experimental}

2.1. Test Equipment. The test equipment mainly used in this research is a six-channel MAST [15], as shown in Figure 1. The MAST is a set of servohydraulic mechanical system controlled by computer. With six electrohydraulic servo actuators in operation, the MAST can provide six-degreeof-freedom motion, which helps to reproduce the vibrating conditions of auto parts.

2.2. Test Procedure. The target spectrum which will be replicated on MAST is an acceleration spectrum collected on a strengthened road of a proving ground. A sensor for road load test was a $3 \mathrm{D}$ accelerometer on the middle position of the upper surface of stack casing. So, the same location point was selected to mount the accelerometer in this paper.

The durability test lasted for $300 \mathrm{~h}$ with a 3D accelerometer installed on the fuel cell stack, during which the response accelerations in the $X-, Y$-, and $Z$-axes directions were collected at regular intervals [16], respectively, $0 \mathrm{~h}, 50 \mathrm{~h}$, $100 \mathrm{~h}, 150 \mathrm{~h}, 200 \mathrm{~h}, 250 \mathrm{~h}$, and $300 \mathrm{~h}$. For each direction, the signal was measured for 3 cycles with the sampling frequency being $256 \mathrm{~Hz}$ and the sampling time being $145 \mathrm{~s}$.

\section{Modeling Approach}

3.1. NARX Neural Network Model. Artificial neural network consists of static neural network and dynamic neural network, in which the current output of the former one is only related to the current input, while that of the later one is related to both the data calculated previously and the current input due to its feedback loop and memory function. Therefore, the dynamic neural network has stronger learning ability and greater nonlinear approximation ability, thus does better in dealing with time series problems.

NARX neural network is a kind of dynamic neural network which uses finite feedback from the output layer to improve the nonlinear approximation ability without much increase in computational cost. Meanwhile, the gradient descent algorithm is very effective in the NARX neural network, which allows the network to converge faster, generalize better, and retain two to three times more information than traditional ones [17]. Thus, it is widely used. The structure of NARX neural network, as can be seen in Figure 2, is usually composed of the input layer, the hidden layer, the output layer, and the delays of the input and output. The defining equation for the NARX model is as follows:

$y(t)=f\left(u(t-1), u(t-2), \cdots, u\left(t-n_{u}\right), y(t-1), y(t-2), \cdots, y\left(t-n_{y}\right)\right)$,

where $y(t)$ is the output of the network at time $t, u(t)$ is the 


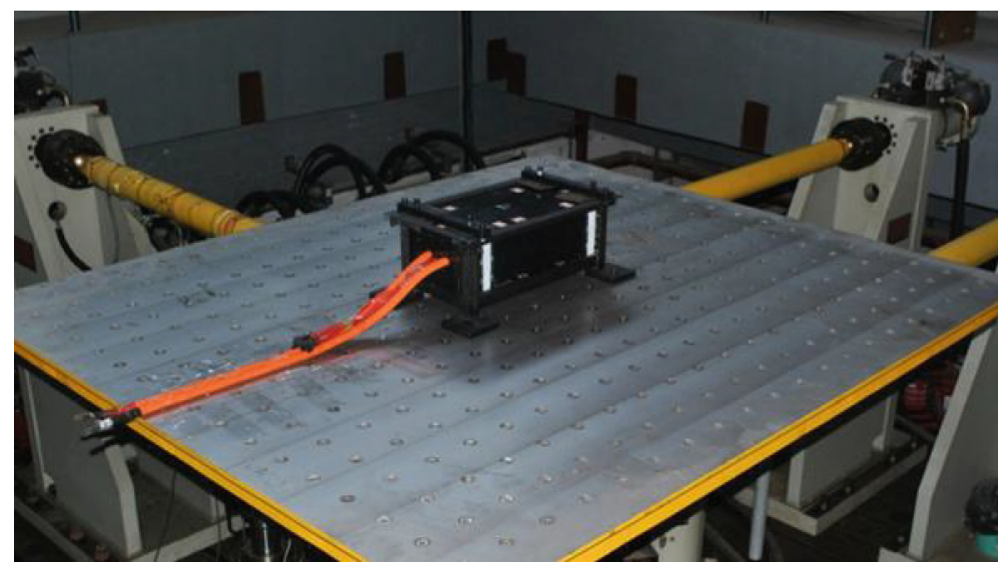

Figure 1: Fuel cell stack fixed on the six-channel MAST.

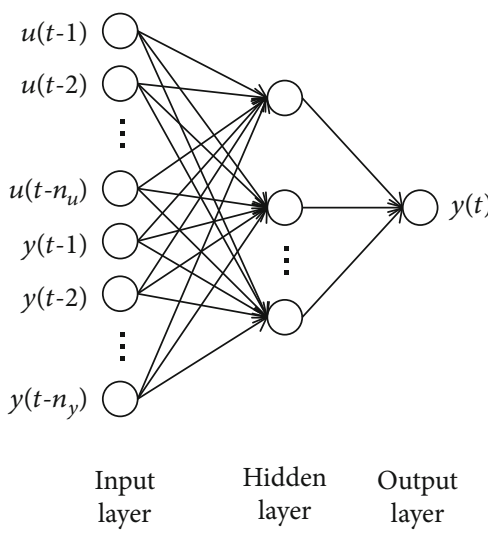

FIGURE 2: Schematic diagram of NARX neural network.

input of the network at time $t, n_{u}$ and $n_{y}$ are, respectively, the delays of the input and output, and $f$ is the nonlinear mapping of the multilayer perceptron network.

There are two modes that can be carried out in training the NARX neural network: the series-parallel (SP) mode and the parallel (P) mode. The difference between these two modes is that the outputs' regression estimators of the network are formed by truth values in the SP mode, while those of the network in the P mode include predicted ones. The SP mode is chosen to train the model in this paper for its faster speed as well as higher accuracy. See Figures 3 and 4.

NARX neural network is widely used in nonlinear system identification, especially for the prediction of nonlinear time series. In this experiment, seven sets of data are collected, each containing nearly 40,000 points in the $X$-, $Y$-, and $Z$ -axes directions, respectively. Thus, it is fit for NARX neural network modeling.

3.2. Data Preprocessing. Data collected by the acquisition system generally contains periodic noise and irregular noise. These two kinds of noise need to be processed before inputting data into the NARX neural network, which helps to restore the data collected to the actual one in real vibrating conditions.

In Section 2, the response signals of the stack were each measured in three cycles at seven different points of time regularly in the $X-, Y$-, and $Z$-axes directions. To ensure the exclusion of the contingency existing in the measurement data, the average value of the response signals of the three periods collected at each time point is obtained as the quasi-response signals in the $X-, Y$-, and $Z$-directions of the time point. However, there are still many glitches and irregular trends in the quasi-response signal, usually caused by noise signals and unexpected interference in testing. The five-spot triple smoothing method is a simple algorithm with good effect used to filter the sampling points, based on least squares polynomial fitting method [18]. Therefore, in the second step, it is used to filter the data, eliminating the interference components without changing the characteristics of the original curve.

3.3. Coherence Analysis. The input of the NARX neural network used in this paper is the vibration excitation signal, and the output is the corresponding response signal. To avoid large prediction errors caused by underfitting of the neural network model, the training set data should include the overall characteristics of the fuel cell stack as much as possible; i.e., the amount of training set data should reach a certain proportion of the total data quantity. By analyzing the coherence of input and output in each direction, whether there is vibration coupling is judged. Thus, the dimension of the input data in each direction is determined.

When the test is carried out on the MAST bench, the fuel cell stack is excited in three directions $(X, Y$, and $Z)$ at the same time, and response signals are collected by a 3-D accelerometer, which indicates the fuel cell stack is a three-input and three-output vibratory system. According to the dimensions of the input data determined above, different strategies are adopted for the input of the model in different directions, thus reduce the computing cost. The coherency function between the excitation and the response can be calculated as follows:

$$
\gamma_{i y}(f)=\frac{\left|G_{i y}(f)\right|^{2}}{G_{i i}(f) G_{y y}(f)},
$$

where $G_{i i}(f)$ and $G_{y y}(f)$ are, respectively, the autopower spectral densities of the excitation and the response and $G_{i y}$ 


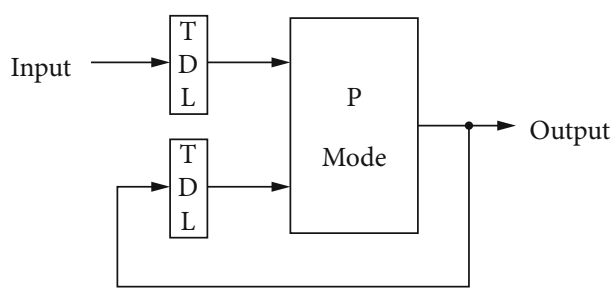

Figure 3: Flow chart of NARX P mode.

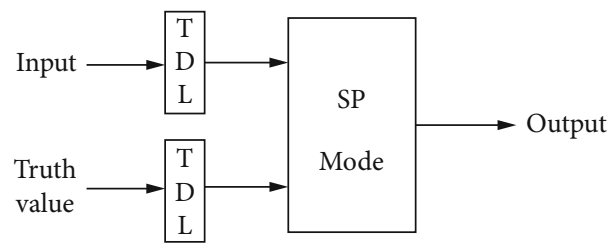

FIGURE 4: Flow chart of NARX SP mode.

$(f)$ is the cross-power spectral density between the excitation and the response.

The coherent coefficients of the excitation in each direction and the response in the $X$-axis direction are shown in Figure 5(a), wherein the correlation coefficient of excitation and response both in the $X$-axis direction is close to 0 in the frequency band outside $20 \mathrm{~Hz}$, while part of that in the frequency band from 1 to $20 \mathrm{~Hz}$ is less than 0.8 . Therefore, the $X$-direction response from 1 to $20 \mathrm{~Hz}$ should consider the influence of excitation in all three directions $(X, Y$, and $Z)$.

Figure 5(b) illustrates the coherent coefficients of the excitation in each direction and the response in the $Y$-axis direction, wherein the correlation coefficient of excitation and response both in the $Y$-axis direction is close to 0 in the frequency band outside $20 \mathrm{~Hz}$, while that in the frequency band from 1 to $20 \mathrm{~Hz}$ is approximately 1 . The other two coherence coefficient diagrams have similar characteristics that they are both relatively small in the whole analysis frequency band. Therefore, the $Y$-direction response from 1 to $20 \mathrm{~Hz}$ is mainly caused by $Y$-direction excitation. Figure 5(c) illustrates the coherent coefficients of the excitation in each direction and the response in the $Z$-axis direction. Similarly, a conclusion can be made that the $Z$ -direction response from 1 to $20 \mathrm{~Hz}$ is mainly caused by $Z$ -direction excitation.

3.4. Model Constructing and Data Grouping. Reasonable extraction of inputs and outputs is necessary for training neural networks. Combined with the analysis above, they can be determined. Taking the $Z$-direction model as an example, the inputs of the network conclude the vibration excitation of the fuel cell stack (i.e., the displacement of the test bench) and the previous outputs' regression estimators both in the $Z$-axis direction, while the outputs of the network are the current $Z$-direction response acceleration of the fuel cell stack. Similarly, the inputs and outputs of the $Y$-direction model correspond to the $Y$-direction data, while those of the $X$-direction model correspond to data in all three directions. In addition, in order to train the neural network more effectively and improve its generalization ability [19], compared with Levenberg-Marquardt algorithm, which converges fast, in this paper, Bayesian regularization algorithm with penalty function is chosen. The number of layers in this network is set to three, in which the activation function of the hidden layer is the sigmoid function, the activation function of the output layer is the linear function, and the number of training iterations is 200 .

In order that the model can fully learn the change rule of the mechanical characteristics (characterized by acceleration) of the system, two sets of data from different training sets and validation sets are set up. The number of delays and neurons in the hidden layer are determined by the method of trial and error. The specific data grouping is shown in Table 1.

\section{Model Validation}

The neural network model will initialize the internal weights and biases. After inputting the training sets in Table 1 into the neural network, the training algorithm will adjust the weights and biases according to the difference between the network output and the expected actual output (i.e., the target) and continuously iterate until the error in the training sets reaches within the range preset. However, this only indicates that the network output of the training sets is accurate. The prediction model focuses on its generalization ability, so it also needs to be validated.

Based on the validation sets, the accuracy of the neural network model can be validated. In this section, the prediction performance of the model is verified by the three methods mentioned in Section 1. The NARX neural network is trained by two groups of training set data in Table 1 . The prediction results will be given below, and the first ones will be taken as an example to carry out correlation test and eigenvalue test.

4.1. Visualization of the Prediction. Vibration signals have different characteristics in time domain and frequency domain. In order to observe the accuracy of the prediction results clearly, the target values and prediction results of the two groups of data in the $X$-, $Y$-, and $Z$-axes directions are given, respectively, in both time domain and frequency domain, as shown in Figure 6.

As can be seen from the first set of prediction results in Figure 6 , the data at $0 \mathrm{~h}, 50 \mathrm{~h}, 100 \mathrm{~h}, 150 \mathrm{~h}, 200 \mathrm{~h}$, and $250 \mathrm{~h}$ are used as training sets to predict the data at $300 \mathrm{~h}$. The prediction results in the three directions are close to the target values and the errors are within the acceptable range. Among them, the results in $Y$ - and $Z$-directions have the best prediction performance. However, the error of the $X$-direction prediction results is slightly larger. By analyzing the original excitation signals, we find that it is caused by some sudden changes of data in the $X$-direction vibration excitation signal. Thanks to the data enhancement method used in the $X$-direction, reasonable results are also obtained. The second set of prediction results in Figure 6 are obtained by using the data at $0 \mathrm{~h}, 50 \mathrm{~h}, 100 \mathrm{~h}, 150 \mathrm{~h}$, and $200 \mathrm{~h}$ as training sets to predict the data at $250 \mathrm{~h}$ and $300 \mathrm{~h}$. Similarly, the prediction results in the three directions also have high accuracy. In 

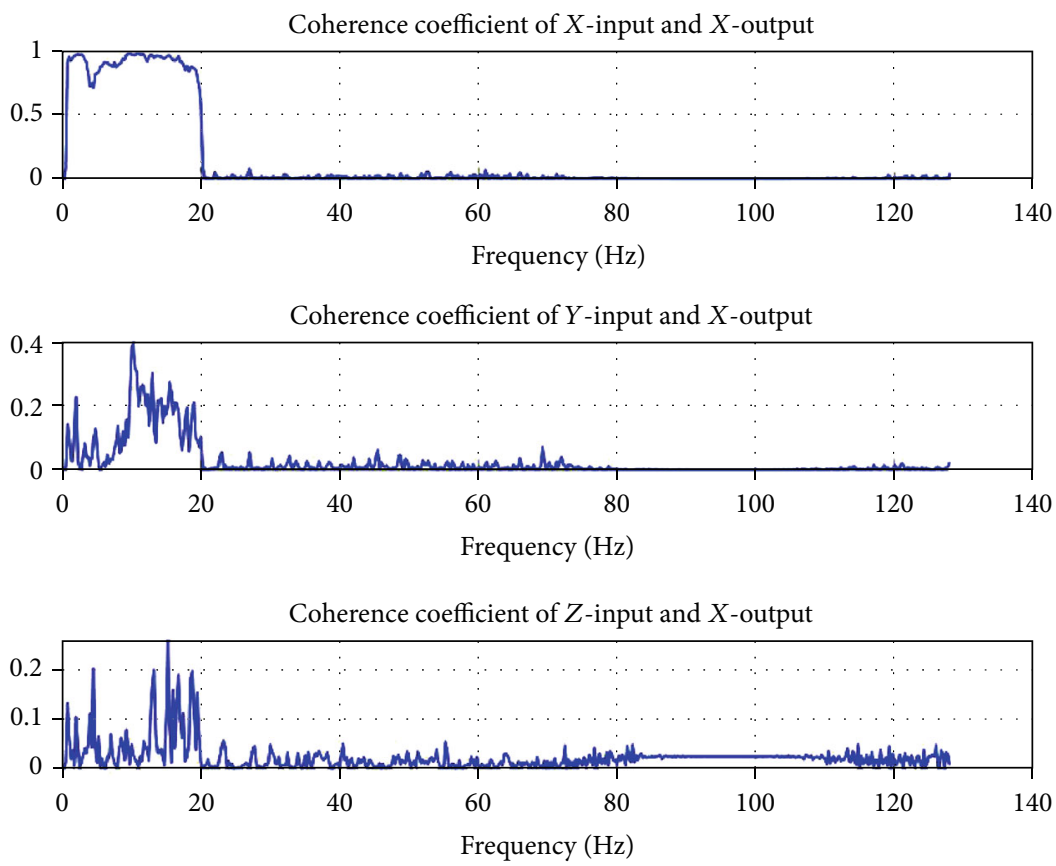

(a) Coherent coefficients between the excitation and the response in the $X$-axis direction
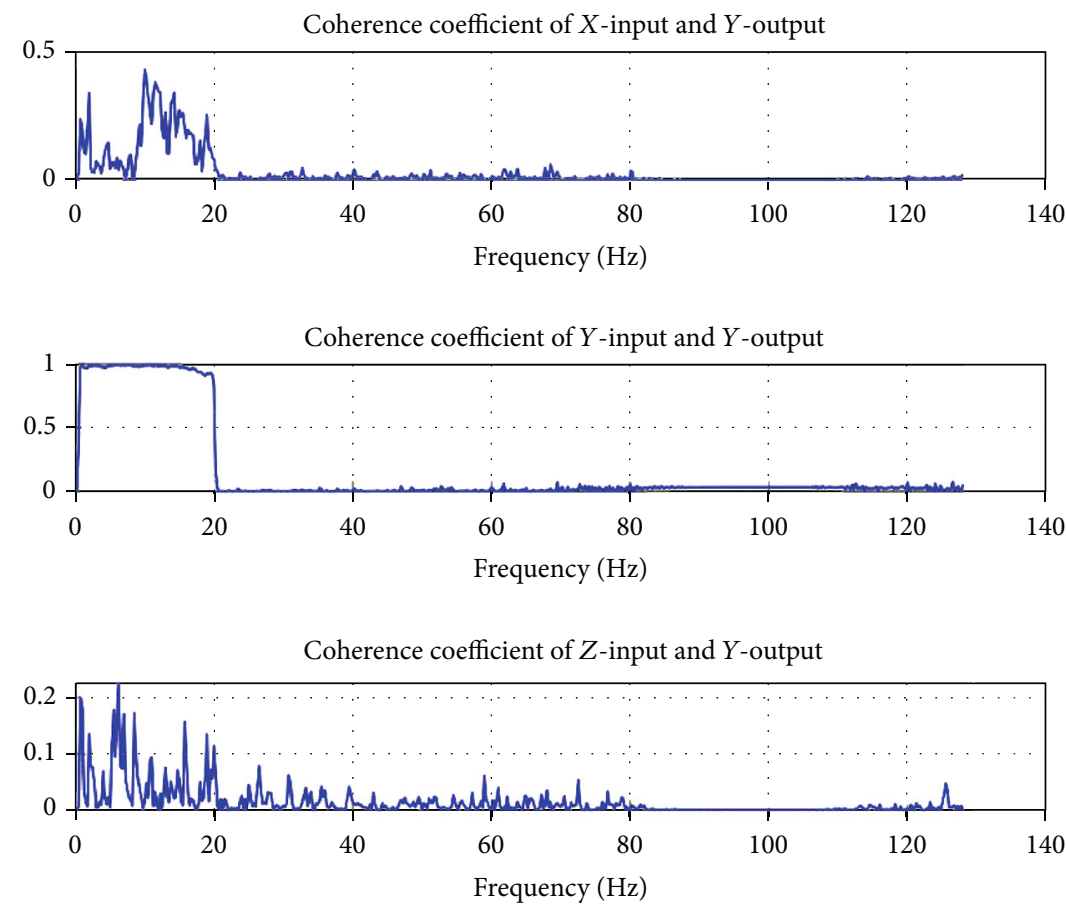

(b) Coherent coefficients between the excitation and the response in the $Y$-axis direction

Figure 5: Continued. 

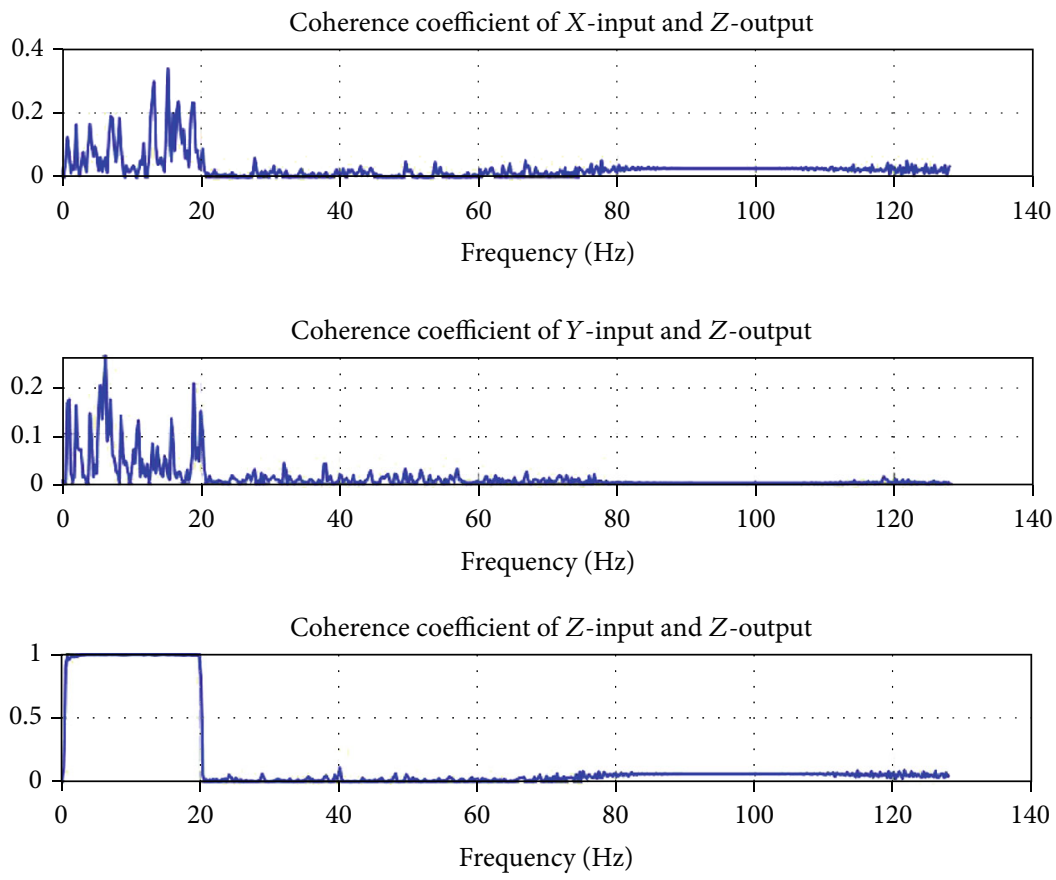

(c) Coherent coefficients between the excitation and the response in the $Z$-axis direction

Figure 5: Coherent coefficients between the excitation and the response at $0 \mathrm{~h}$.

TABLE 1: Data grouping.

\begin{tabular}{lcccr}
\hline Sets & Training sets & Validation sets & Delay order & Number of neurons in hidden layer \\
\hline Set I & $0 \mathrm{~h}, 50 \mathrm{~h}, 100 \mathrm{~h}, 150 \mathrm{~h}, 200 \mathrm{~h}, 250 \mathrm{~h}$ & $300 \mathrm{~h}$ & 5 & 15 \\
Set II & $0 \mathrm{~h}, 50 \mathrm{~h}, 100 \mathrm{~h}, 150 \mathrm{~h}, 200 \mathrm{~h}$ & $250 \mathrm{~h}, 300 \mathrm{~h}$ & 5 & 15 \\
\hline
\end{tabular}

the time domain, the amplitude of the predicted value and the target value are similar, and the curves almost overlap. In the frequency domain, the characteristic frequency prediction is accurate in the main vibration frequency range from 1 to $20 \mathrm{~Hz}$.

Comprehensively analyzing the prediction results above, it can be preliminarily judged that the model has fairly good accuracy and generalization ability.

4.2. Correlation Test. However, it is incomplete to only observe the prediction results in Figure 6. For the prediction model of time series data, the correlation test is required. The prediction error in the validation sets is expressed as follows:

$$
\varepsilon(t)=y(t)-\widehat{y}(t)
$$

where $\varepsilon(t)$ is the prediction error and $y(t)$ and $\hat{y}(t)$ are, respectively, the target value and the network outputs of the response signal.

An ideal fuel cell vibration model should contain all dynamic information of the whole system and have the following two characteristics [13]:

(1) The prediction error of its prediction results should be uncorrelated between different time points
(2) The prediction error and the input data should be uncorrelated

The accuracy of the model can be verified by calculating the autocorrelation coefficient of $\varepsilon(t)$ and the crosscorrelation coefficient between the inputs and $\varepsilon(t)$. Taking the prediction results of the first group of data in Table 1 as an example, the above two correlation coefficients are analyzed, and the results are shown in Figure 7. As displayed in Figure 7, the characteristics of the autocorrelation coefficients of $\varepsilon(t)$ in the three directions are similar, where the value reaches to the maximum when the value of lag is 0 , then decreasing rapidly to around 0 . It shows that the prediction error of each data point is independent of each other. In addition, the cross-correlation coefficients are mostly within the confidence intervals indicated by the red lines in the figure, which means that the prediction error is also independent of the inputs. These two features reflected in the correlation image, respectively, correspond to the two characteristics of the above ideal fuel cell vibration model, which verifies the model's high accuracy.

4.3. Eigenvalue Test. Vibration signals have certain eigenvalues, of which the maximum and root mean square (RMS) values in both time domain and frequency domain and the total power of the signals can comprehensively reflect 

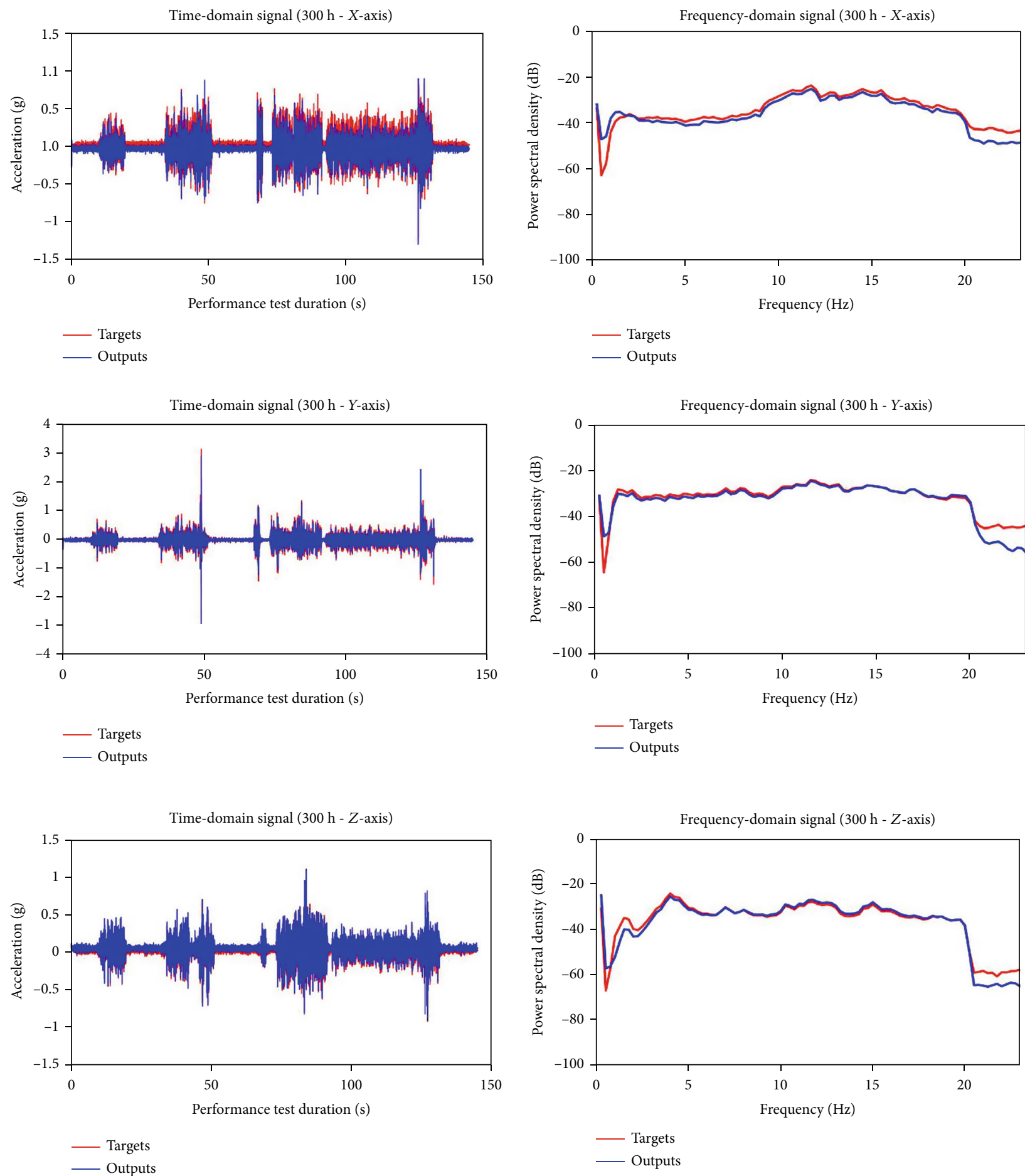

(a) Set I

FIgURE 6: Continued. 

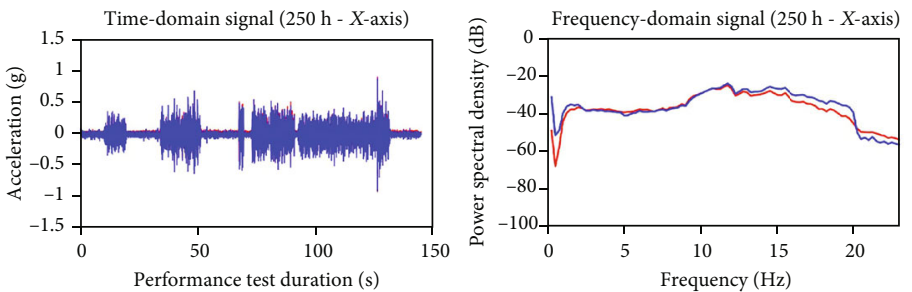

$$
\begin{aligned}
& \text { Targets } \\
& \text { - Outputs }
\end{aligned}
$$

$$
\begin{aligned}
& \text { Targets } \\
& \text { Outputs }
\end{aligned}
$$
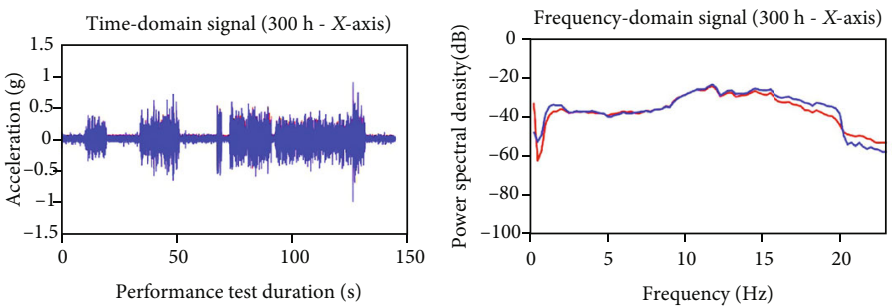

— Targets

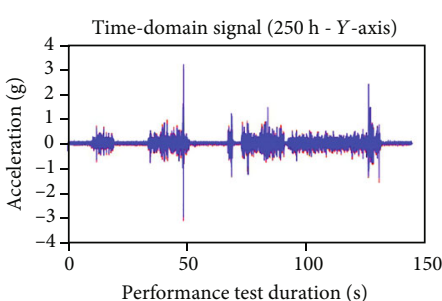

— Target

$$
\text { —_ Outputs }
$$

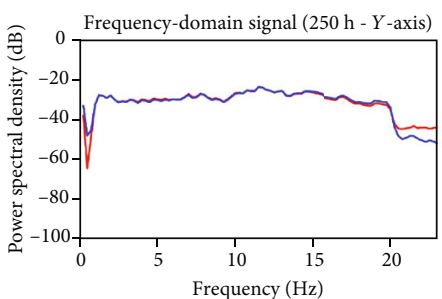

— Targets

_ Targets

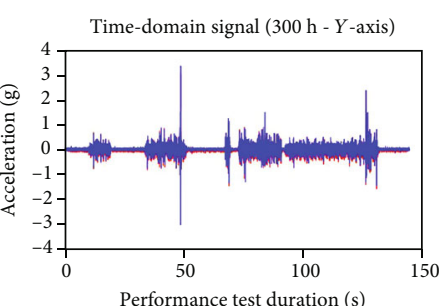$$
\text { - Outputs }
$$

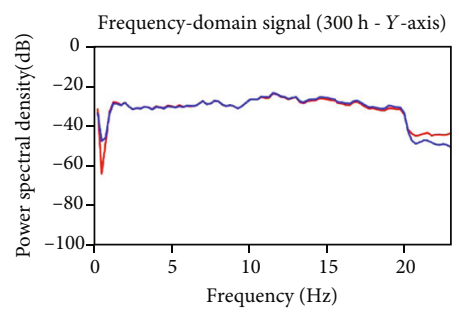

$$
\begin{aligned}
& \text { Targets } \\
& \text { O Outputs }
\end{aligned}
$$

$$
\begin{aligned}
& \text { Targets } \\
& \text { Outputs }
\end{aligned}
$$
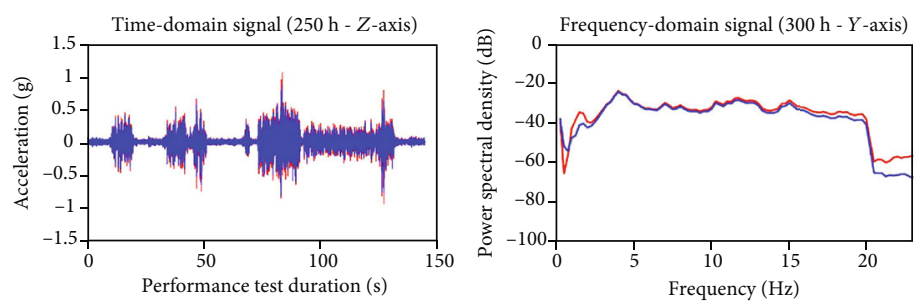

$$
\text { Targets }
$$

$$
\begin{aligned}
& \text { Targets } \\
& \text { _ Outputs }
\end{aligned}
$$
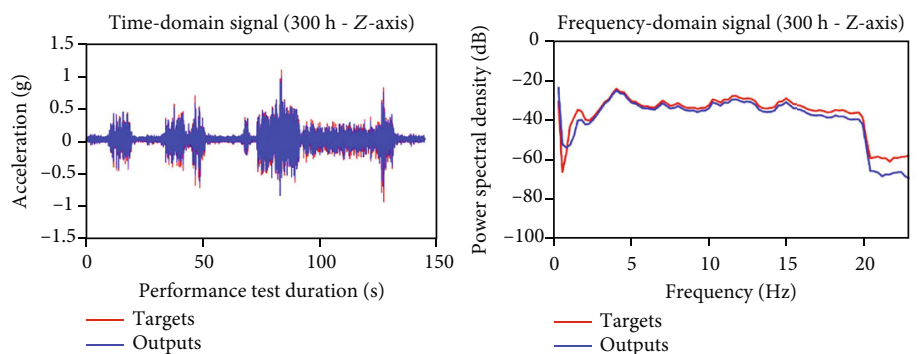

(b) Set II

Figure 6: Contrast diagrams for network outputs and target values in both time domain and frequency domain. 

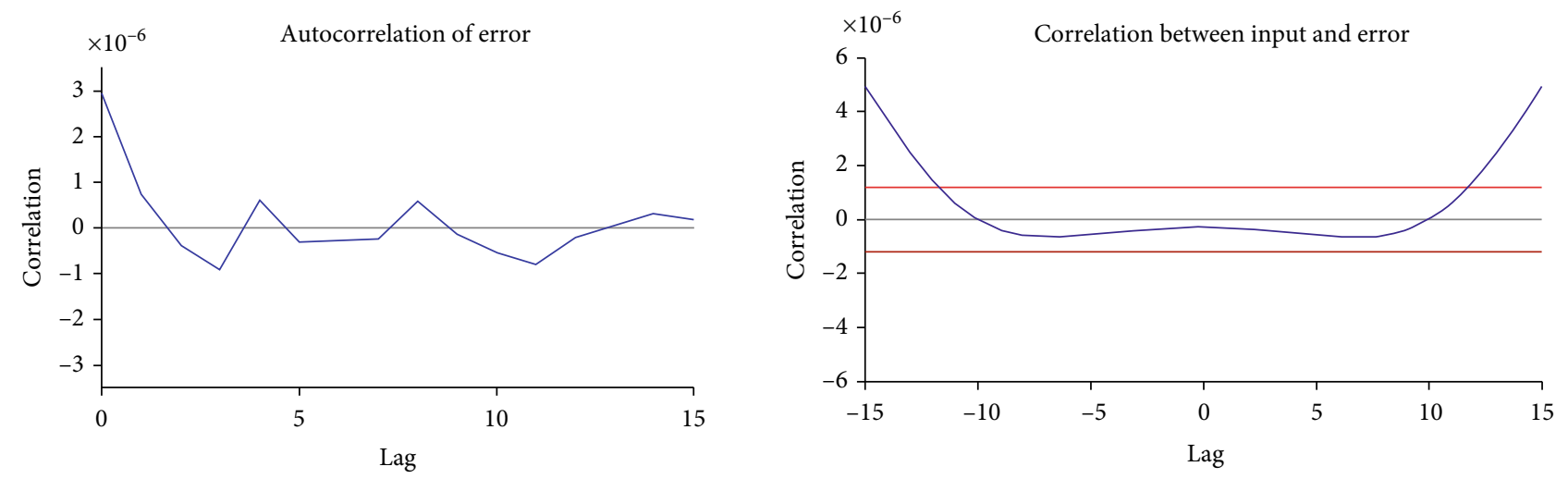

(a) $X$-direction
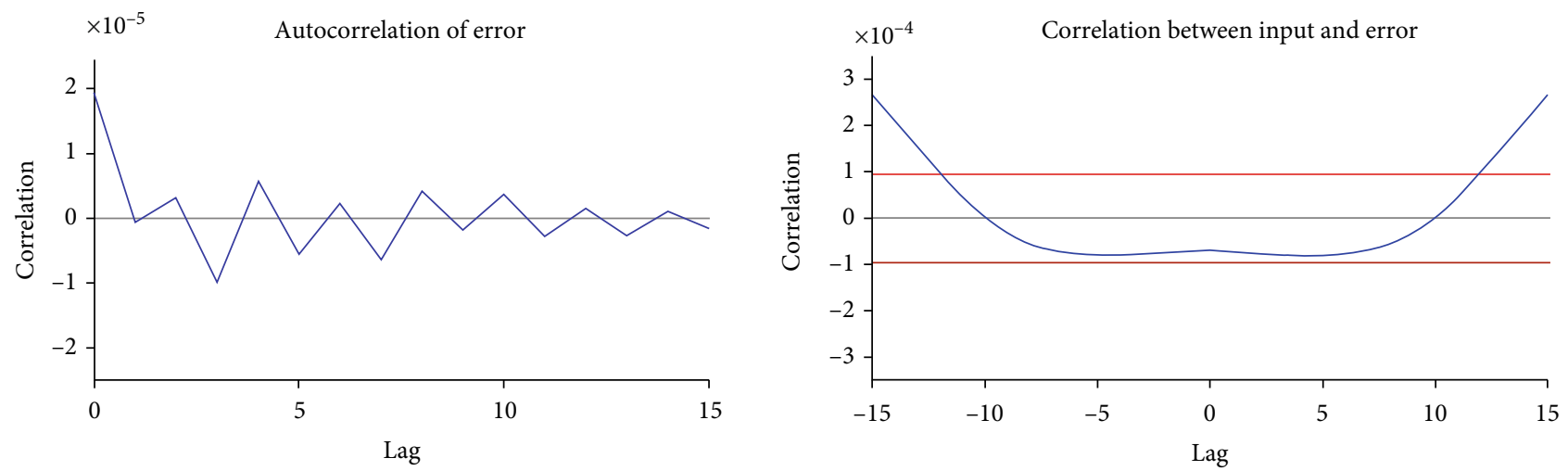

(b) $Y$-direction
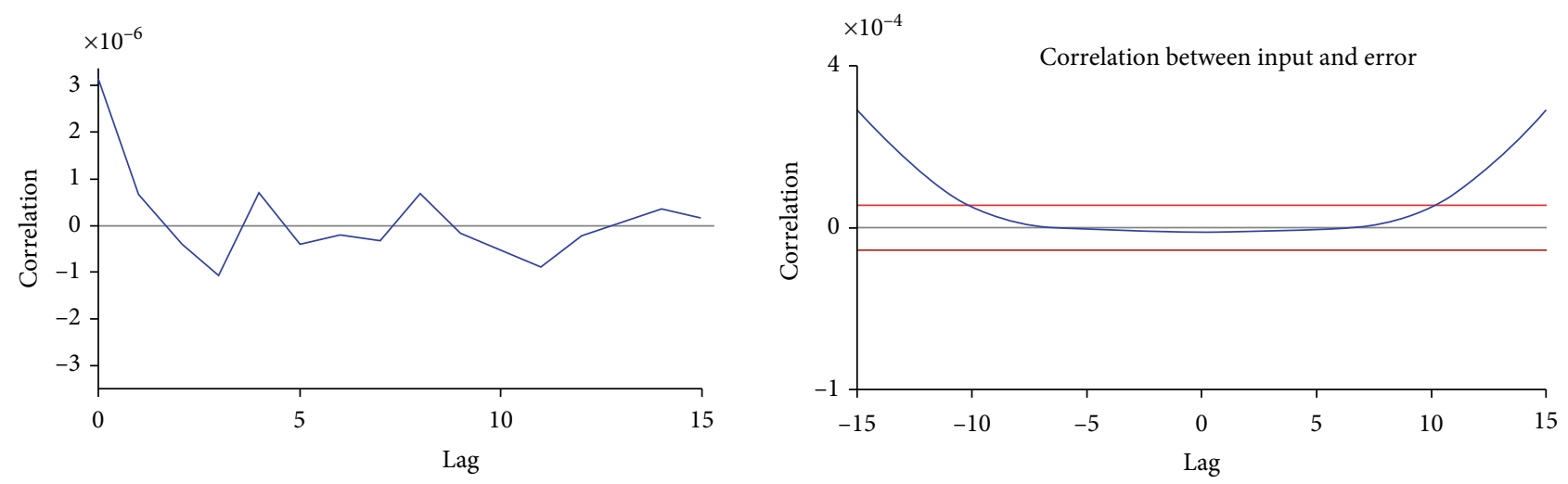

(c) Z-direction

FIgURE 7: Correlation coefficient.

the characteristics. From these five eigenvalues, the error analysis of the data in the validation sets is carried out in an intuitive way. The target value, the network output value, and the absolute relative error of the eigenvalues at $300 \mathrm{~h}$ in $X$-, $Y$-, and $Z$-directions are given in Table 2, from which it can be found that the prediction results in the three directions have high accuracy overall. For the network output values in $X$-direction, except that the maximum error in the time domain exceeds $10 \%$, the accuracy of other eigenvalues is about $3 \%$. The prediction performance in $Y$-direction is of the highest accuracy, with 5 eigenvalues all around 3\%. In $Z$ -direction, the error of RMS in time domain and frequency domain and the total signal power is close to $10 \%$, while the error of maximum value in both time domain and frequency domain is very small.

4.4. Discussions. The model is verified by the above three methods, based on the validation sets in $X$-, $Y$-, and $Z$-directions. To make a comprehensive analysis, conclusions can be made that the prediction performances in different directions differ slightly due to the acceleration response characteristics of their own, but all within an acceptable range, of which the $Y$-axis data has the highest prediction accuracy. In the meantime, this model is of good stability and generalization. 
TABLE 2: The contrast of the eigenvalues.

\begin{tabular}{|c|c|c|c|c|c|c|c|c|c|}
\hline \multirow[t]{2}{*}{ Eigenvalues } & \multicolumn{3}{|c|}{ Target values } & \multicolumn{3}{|c|}{ Output values } & \multicolumn{3}{|c|}{$\begin{array}{l}\text { Absolute value of relative } \\
\text { error }\end{array}$} \\
\hline & $X$ & $Y$ & $Z$ & $X$ & Y & $Z$ & $X$ & Y & Z \\
\hline Maximum in time domain (g) & 1.167 & 3.128 & 1.117 & $9.93 \mathrm{E}-1$ & 3.069 & 1.106 & $14.90 \%$ & $1.89 \%$ & $0.98 \%$ \\
\hline RMS in time domain (g) & $1.399 E-1$ & $1.69 E-1$ & $1.20 E-1$ & $1.37 E-1$ & $1.65 E-1$ & $1.32 E-1$ & $2.22 \%$ & $2.83 \%$ & $9.65 \%$ \\
\hline Maximum in frequency domain $(\mathrm{g})$ & $4.308 E-3$ & $4.19 E-3$ & $3.79 E-3$ & $4.17 E-3$ & $4.05 E-3$ & $3.99 E-3$ & $3.30 \%$ & $3.46 \%$ & $5.33 \%$ \\
\hline RMS in frequency domain $(\mathrm{g})$ & $5.397 E-4$ & $6.31 E-4$ & $3.70 E-4$ & $5.23 E-4$ & $6.13 E-4$ & $4.16 E-4$ & $3.13 \%$ & $2.82 \%$ & $12.50 \%$ \\
\hline Total signal power $\left(\mathrm{g}^{2}\right)$ & $1.876 E-2$ & $2.78 E-2$ & $1.40 E-2$ & $1.82 E-2$ & $2.70 E-2$ & $1.56 E-2$ & $3.09 \%$ & $2.87 \%$ & $11.11 \%$ \\
\hline
\end{tabular}

\section{Conclusion}

In this paper, a fuel cell stack vibration prediction model based on NARX neural network is established. And the model is trained and verified by using two sets of vibration durability testing data. Summing up the full text, the following conclusions can be drawn:

(1) The vibration prediction model of fuel cell stack based on NARX neural network is suitable for durability testing data with strong nonlinearity and can predict the variations in vibration characteristics of fuel cell stack

(2) This model has high prediction accuracy. The data of the verification sets have high consistency between the network outputs and the target values in the time domain and frequency domain images, the correlation coefficient is within the confidence interval, and the error of eigenvalue is mostly within $10 \%$

(3) The prediction model has high stability and generalization ability. The time and frequency domain prediction results of the two validation sets all reflect high consistency in $X-, Y$-, and $Z$-directions

\section{Data Availability}

The raw data required to reproduce these findings cannot be shared at this time as the data also forms part of an ongoing study.

\section{Conflicts of Interest}

The authors declare that there are no conflicts of interest regarding the publication of this paper.

\section{Acknowledgments}

The work was financially supported by the Shanghai Science and Technology Committee (Project Number: 19DZ1206401).

\section{References}

[1] US Department of Energy, "Hydrogen and fuel cells program overview," http://www.eere.energy.gov/hydrogenandfuelcells/ presentations.html.
[2] M. C. Bétournay, G. Bonnell, E. Edwardson, D. Paktunc, A. Kaufman, and A. T. Lomma, "The effects of mine conditions on the performance of a PEM fuel cell," Journal of Power Sources, vol. 134, no. 1, pp. 80-87, 2004.

[3] B. Liu, L. F. Liu, M. Y. Wei, and C. W. Wu, "Vibration mode analysis of the proton exchange membrane fuel cell stack," Journal of Power Sources, vol. 331, pp. 299-307, 2016.

[4] S. Khatir, T. Khatir, D. Boutchicha et al., "An efficient hybrid TLBO-PSO-ANN for fast damage identification in steel beam structures using IGA," Smart Structures and Systems, vol. 25, no. 5, pp. 605-617, 2020.

[5] H. Tran-Ngoc, S. Khatir, T. le-Xuan, G. de Roeck, T. Bui-Tien, and M. Abdel Wahab, "A novel machine-learning based on the global search techniques using vectorized data for damage detection in structures," International Journal of Engineering Science, vol. 157, article 103376, 2020.

[6] H. Tran-Ngoc, S. Khatir, H. Ho-Khac, G. de Roeck, T. Bui-Tien, and M. Abdel Wahab, "Efficient artificial neural networks based on a hybrid metaheuristic optimization algorithm for damage detection in laminated composite structures," Composite Structures, vol. 262, article 113339, 2021.

[7] S. Khatir, D. Boutchicha, C. le Thanh, H. Tran-Ngoc, T. N. Nguyen, and M. Abdel-Wahab, "Improved ANN technique combined with Jaya algorithm for crack identification in plates using XIGA and experimental analysis," Theoretical and Applied Fracture Mechanics, vol. 107, article 102554, 2020.

[8] R. Zenzen, S. Khatir, I. Belaidi, C. le Thanh, and M. Abdel Wahab, "A modified transmissibility indicator and artificial neural network for damage identification and quantification in laminated composite structures," Composite Structures, vol. 248, article 112497, 2020.

[9] J. M. P. Menezes and G. A. Barreto, "Long-term time series prediction with the NARX network: an empirical evaluation," Neurocomputing, vol. 71, no. 16-18, pp. 3335-3343, 2008.

[10] S. R. Devi, P. Arulmozhivarman, C. Venkatesh, and P. Agarwal, "Performance comparison of artificial neural network models for daily rainfall prediction," International Journal of Automation and Computing, vol. 13, no. 5, pp. 417-427, 2016.

[11] V. Rouss and W. Charon, "Multi-input and multi-output neural model of the mechanical nonlinear behaviour of a PEM fuel cell system," Journal of Power Sources, vol. 175, no. 1, pp. 1-17, 2008.

[12] V. Rouss, P. Lesage, S. Begot et al., "Mechanical behaviour of a fuel cell stack under vibrating conditions linked to aircraft applications part I: experimental," International Journal of Hydrogen Energy, vol. 33, no. 22, pp. 6755-6765, 2008. 
[13] V. Rouss, D. Candusso, and W. Charon, "Mechanical behaviour of a fuel cell stack under vibrating conditions linked to aircraft applications part II: three-dimensional modelling," International Journal of Hydrogen Energy, vol. 33, no. 21, pp. 6281-6288, 2008.

[14] V. Rouss, W. Charon, and A. Desflots, "Characterisation of mechanical non-linearities in a proton exchange membrane fuel cell using raw data," International Journal of Hydrogen Energy, vol. 34, no. 5, pp. 2377-2386, 2009.

[15] "MTS Systems Corporation. Standard Multi-Axial Simulation Table (MAST) TM Systems," http://www.mts.com/en/ products/producttype/test-systems/simulationsystems/ multiaxial-simulation-tables/orthogonal/index.htm.

[16] Y. Hou, W. Zhou, and C. Shen, "Experimental investigation of gas-tightness and electrical insulation of fuel cell stack under strengthened road vibrating conditions," International Journal of Hydrogen Energy, vol. 36, no. 21, pp. 13763-13768, 2011.

[17] Tsungnan Lin, B. G. Horne, P. Tino, and C. L. Giles, "Learning long-term dependencies in NARX recurrent neural networks," IEEE Transactions on Neural Networks, vol. 7, no. 6, pp. 13291338, 1996.

[18] W. U. Wei, C. Biao, W. U. Jianfeng, and H. Kui, "Study on reverse deduction of reservoir-inflow based on cubical smoothing algorithm with five-point approximation," Water Resources and Hydropower Engineering, vol. 44, 2013.

[19] D. Niu, L. Ji, M. Xing, and J. Wang, "Multi-variable echo state network optimized by Bayesian regulation for daily peak load forecasting," Journal of Networks, vol. 7, no. 11, 2012. 